\title{
Possible deviations from Griffith's criterion in shallow slabs, and consequences on slab avalanche release
}

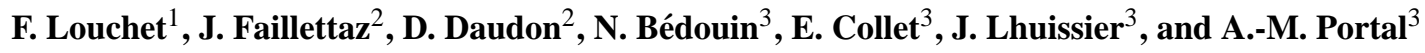 \\ ${ }^{1}$ LTPCM - UMR, CNRS 5614/INPG-UJF, B.P. 75, F-38402 St. Martin d'Hères, France \\ ${ }^{2}$ Sols, Solides, Structures, ENSHMG, B.P. 75, F-38402 St. Martin d'Hères, France \\ ${ }^{3}$ ENSEEG, B.P. 75, F-38402 St. Martin d'Hères, France
}

Received: 20 September 2001 - Revised: 10 January 2002 - Accepted: 11 January 2002

\begin{abstract}
Possible reasons for deviations from Griffith's criterion in slab avalanche triggerings are examined. In the case of a major basal crack, we show (i) that the usual form of Griffith's criterion is valid if elastic energy is stored in a shallow and hard slab only, and (ii) that rapid healing of broken ice bonds may lead to shear toughnesses larger than expected from tensile toughness experiments. In the case of avalanches resulting from failure of multi-cracked weak layers, where a simple Griffith's criterion cannot be applied, frequency/size plots obtained from discrete elements and cellular automata simulations are shown to obey scale invariant power law distributions. These findings are confirmed by both frequency/acoustic emission duration and frequency/size plots obtained from field data, suggesting that avalanche triggerings may be described using the formalism of critical phenomena.
\end{abstract}

\section{Introduction}

It is widely accepted that slab avalanche release results from the propagation of a basal crack, followed by a crown crack opening. The stability of a crack in a bulk solid is classically determined by the so-called Griffith's criterion $\tau \sqrt{\pi a}=K_{c}$, where $\tau$ is the applied stress, $a$ is the crack size, and $K_{c}$ is the material toughness. This expression results from a balance between the release rate of the stored elastic energy and the work required to create new surfaces that allow further propagation of the crack.

However, the application of Griffith's approach to avalanche release is not straightforward, due to at least four factors. The first one is that toughness data are poorly documented in snow: there is a factor of about 20 between theoretical and experimental estimates that leads to a factor 400 in crack critical size. The second is that Griffith's criterion is, in principle, valid if the elastic energy is stored in a three-

Correspondence to: F. Louchet

(francois.louchet@ltpcm.inpg.fr) dimensional volume of matter. Since the amount of elastic energy stored per unit volume is $\tau^{2} / 2 E$, where $E$ is the material Young's modulus, softer matter stores more elastic energy than stiffer matter. Griffith's criterion may thus apply without any modification to either basal or crown cracks in the case of a relatively thick snow slab deposited on a thick and relatively soft snow substrate, but some corrections are expected to be necessary in the case of a basal crack located between a shallow and soft slab and a stiff substrate. The third factor, developed in Sect. 2.1, is that basal crack surfaces may experience frictional stresses that may significantly decrease stress concentrations at the crack tip, which is equivalent to an apparent increase in the shear toughness $K_{I I C}$. The fourth factor is that Griffith's criterion deals with the stability of a single crack, whereas avalanche release, and in particular, natural avalanche release, is more likely to result from a catastrophic evolution of a large number of small, elastically interacting basal defects, rather than from that of a single large crack.

The goal of the present paper is to discuss the possible reasons for the inconsistency of toughness data, and to explore both the validity limits of Griffith's criterion in the case of shallow slabs, and a possible application to avalanche release of methods derived from the theory of critical phenomena in statistical physics.

\section{Single defect}

\subsection{Influence of friction}

Current observation shows that many human triggered avalanches start from tens or hundreds of meters above the skier, which suggests that they are triggered by a rapid extension of a basal crack. According to Griffith's criterion, such an instability of the basal crack takes place for a critical crack size $a_{s}$ given by:

$\tau \sqrt{\pi a s_{s}}=K_{I I C}$, 


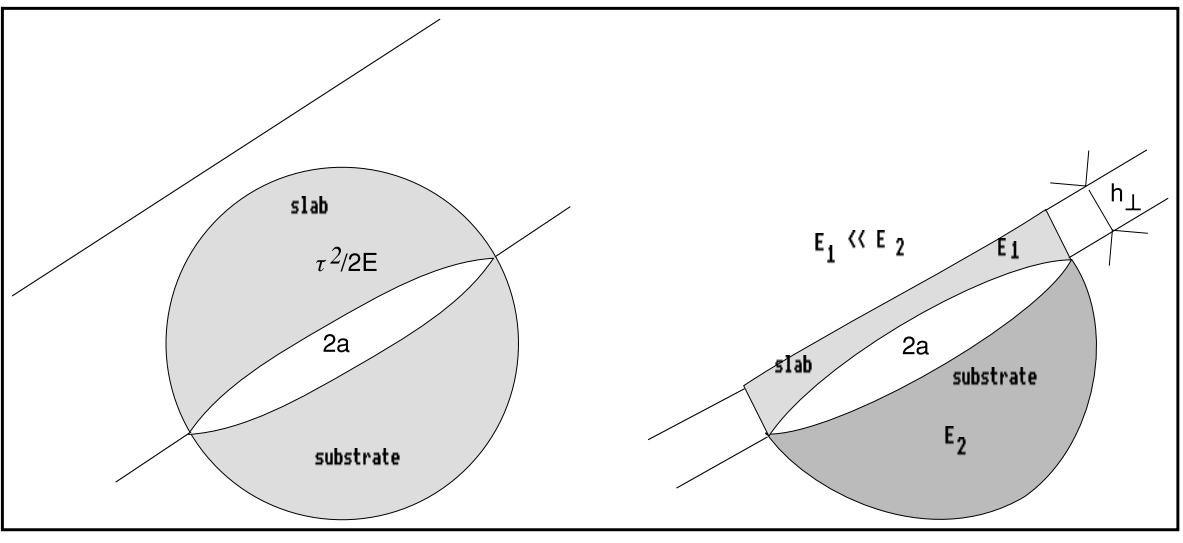

Fig. 1. Schematic spatial distribution of stored elastic energy around a basal crack: (a) in the case of a deep slab, and (b) in the case of a shallow slab of depth $h_{\perp}$ where $K_{I I C}$ is the weak layer toughness in mode II (i.e. in shear), and where the shear stress $\tau$ is related to slope $a$, snow density $\rho$, and slab depth $h$ (measured vertically) (1) by:

$\tau=\frac{\rho g h}{2} \sin 2 \alpha$.

From Eqs. (1) and (2), the critical crack size is:

$a_{s}=\frac{1}{\pi}\left(\frac{2 K_{I I C}}{\rho g h \sin 2 \alpha}\right)^{2}$.

Taking a $K_{I I c}$ value of $1.7210^{-2} \mathrm{MPa} \sqrt{m}$, which is a theoretical estimate computed from Gibson and Ashby (1987) for a snow density of $400 \mathrm{~kg} / \mathrm{m}^{3}$, a slope of $40^{\circ}$, and a slab depth of $1 \mathrm{~m}$, one finds a critical size of a few tens of meters that does not seem unreasonable, if compared to the average distance travelled by a skier on a slab before avalanche triggering.

One can also use an experimental value of $K_{I I c}$, instead of the theoretical value taken above: in classical materials, $K_{I I c}$ can be computed from, and is of the same order of magnitude as the toughness in mode I (tension) $K_{I c}$. The only value of $K_{I c}$ available in the literature can be found in Kirchner et al. (2000). For a snow density of $400 \mathrm{~kg} / \mathrm{m}^{3}$, $K_{I c} \approx 1 \mathrm{kPa} \sqrt{m}$ gives, under the same conditions as above, a critical crack size of the order of a few $\mathrm{cm}$, i.e. not far from the size of natural heterogeneities. This would mean that such a slab should be intrinsically unstable, or in other words, that the slab depth corresponding to a critical crack size of a few tens of meters as in the previous example, should only be of $6 \mathrm{~cm}$, which is not likely to be true.

A possible reason for this discrepancy may be that in real life, stress concentrations at the crack tip may be significantly reduced by frictional stresses between both crack surfaces. If Coulomb's law is assumed to be valid in the present case, such a friction stress may be taken proportional to the normal stress, which gives, after simple calculations, a critical size Louchet (2001):

$a_{s}=\frac{K_{C}}{\pi(\rho g h)^{2} \cos ^{2} \alpha(\sin \alpha-A \cos \alpha)^{2}}$,

where $A$ is the friction coefficient $(0<A<1)$. Taking $K_{I I c} \approx K_{I c} \approx 1 \mathrm{kPa} \sqrt{m}, a=40^{\circ}$ and $h=1 \mathrm{~m}$, the friction coefficient that would give a critical crack size $a_{s} \approx 10 \mathrm{~m}$ is $A=0.83$, which is far from being negligible.

This result is in agreement with the fact that basal crack surfaces are not perfectly planar, and the existence of compression zones may considerably reduce stress concentrations at the crack tip. It also agrees with the idea that damaged snow may heal in a relatively short time during the shear process, through rewelding of broken ice bonds. A kinetic approach of snow creep instabilities under shear loading (Louchet, 2000), based on such a balance between ice bond breaking and rewelding, leads to a specific kinetic definition of $K_{I I c}$, which has the same dimensionality and is a generalisation of the static definition of $K_{I I c}$. In contrast with mode I (tensile) toughness $K_{I c}$, in which ice bonds fail but cannot reconstruct, this kinetic definition of shear toughness $K_{I I c}$ that involves damage and healing mechanisms is expected to lead to values significantly larger than those deduced from $K_{I c}$ using elasticity calculations. This specific microscopically-based shear toughness may be considered as qualitatively equivalent to the macroscopic friction coefficient introduced above.

\subsection{Influence of dimensionality}

Another limitation of Griffith's approach is that elastic energy may not necessarily be stored in a 3-D volume. Let us consider as a first approximation that the elastic energy associated with the basal crack is stored in both a slab of depth $h_{\perp}$ (measured perpendicular to the slope; Fig. 1) and of Young's modulus $E_{1}$, and a half spherical zone in the old snow substrate of Young's modulus $E_{2}$.

Griffith's criterion is based on the idea that a crack becomes unstable when the surface energy required to increase the crack radius of $d a$ is balanced by a corresponding amount of relaxed elastic energy, which can be written in the present case:

$\frac{d}{d a}\left(\frac{\tau^{2}}{2 E_{1}} \pi a^{2} h_{\perp}+\frac{\tau^{2}}{2 E_{2}} \frac{2}{3} \pi a^{3}\right) d a \approx 2 \gamma_{s} 2 \pi a d a$,

in which the bracket represents the stored elastic energy, $a$ is the basal crack radius, and $\gamma_{s}$ is the specific surface energy. 


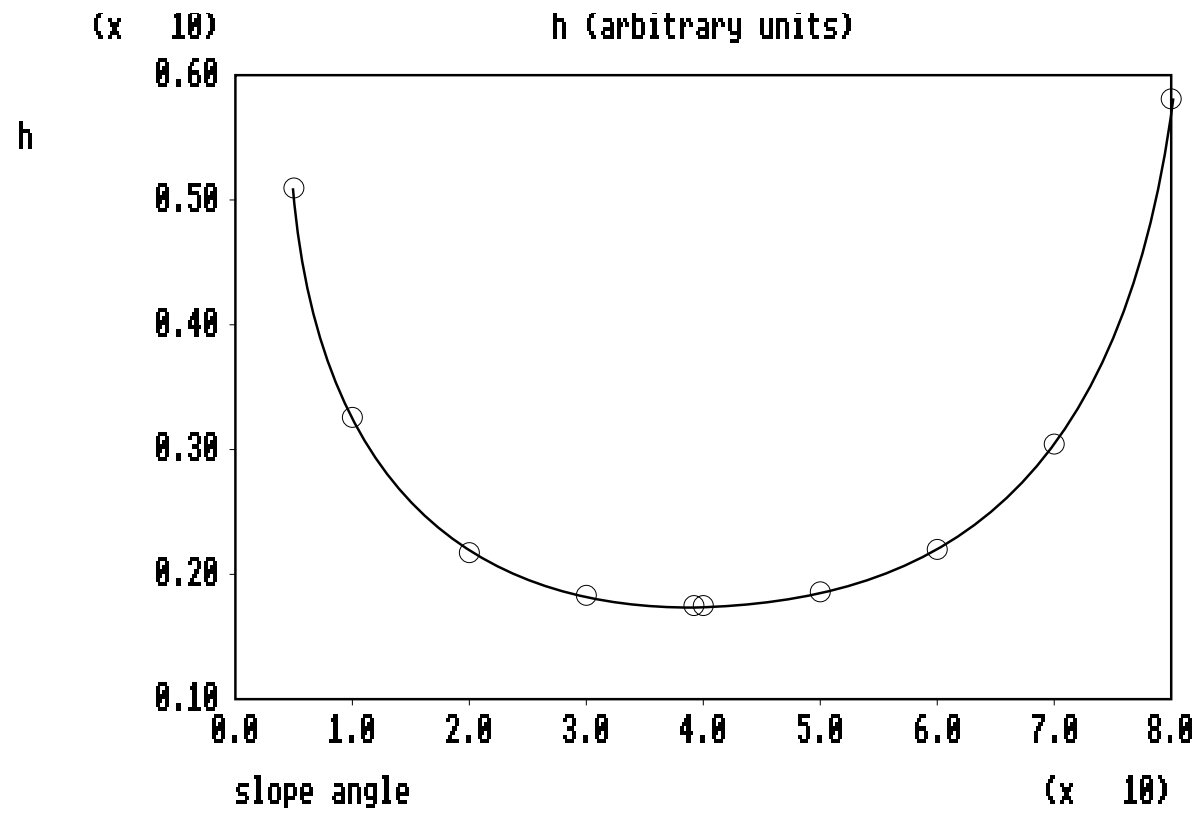

Fig. 2. Variations with slope of the critical slab depth $h$ for avalanche release in the limiting case of a soft shallow slab.
Since $h_{\perp}=h \cos \alpha$, it can be seen from Eqs. (1) and (5) that the critical crack size $a_{s}$ satisfies:

$\left(\frac{\rho g h}{2} \sin 2 \alpha\right)^{2}\left(\frac{h \cos \alpha}{E_{1}}+\frac{a_{s}}{E_{2}}\right)=4 \gamma_{s}$.

Two limiting cases can be considered:

- Hard and shallow slab on soft substrate. In this case, $h / E_{1} \ll a_{s} / E_{2}$ and the first term in the bracket in Eq. (6) is small as compared to the second one (significant elastic energy stored in the substrate). The critical basal crack size is given by:

$$
\begin{aligned}
& a_{s}=a_{s}^{0}-h \cos \alpha \frac{E_{2}}{E_{1}}=\frac{1}{\pi}\left(\frac{2 K_{I I c}}{\rho g h \sin 2 \alpha}\right)^{2} \\
& -h \cos \alpha \frac{E_{2}}{E_{1}}<a_{s}^{0},
\end{aligned}
$$

where $a_{s}^{0}$ is the classical Griffith's critical crack size. The correction term $-h \cos \alpha\left(E_{2} / E 1\right)$ is negative, which means that the avalanche is released for smaller crack sizes than in the classical situation. This result can be equivalently written in terms of toughness:

$$
\tau \sqrt{\pi a_{s}} \approx \sqrt{4 E_{2} \gamma_{s}}\left(1-\frac{1}{2}\left(\frac{h_{\perp}}{E_{1}}\right) \frac{\tau^{2}}{4 \gamma_{s}}\right) .
$$

- Soft and relatively thick slab on hard substrate. Now $h / E_{1} \ll a_{s} / E_{2}$, which means that the energy is mainly stored in the slab. Equation (6) may be written in a form equivalent to Eq. (8):

$$
\tau \sqrt{\pi h_{\perp}} \approx \sqrt{4 \pi E_{1} \gamma_{s}}\left(1-\frac{1}{2}\left(\frac{a_{s}}{E_{2}}\right) \frac{\tau^{2}}{4 \gamma_{s}}\right),
$$

where the "stress concentration factor" is now $\tau \sqrt{\pi h_{\perp}}$ instead of $\tau \sqrt{\pi a}$. This equation takes a form similar to Griffith's criterion, but where the crack size $a$ is replaced by the slab depth $h_{\perp}$. The correction factor is negative, as in Eqs. (7) or (8). When the correction factor becomes negligible, the crack instability criterion becomes independent of crack size. This result can be equivalently written:

$$
\begin{aligned}
& h \approx\left(\frac{4 E_{1} \gamma_{s}}{(\rho g)^{2}} \frac{1}{\sin ^{2} \alpha \cos ^{3} \alpha}\right)^{1 / 3} \\
& \approx\left(\frac{K_{I I c}}{\rho g}\right)^{2 / 3} \frac{1}{\sin ^{2 / 3} \alpha \cos \alpha},
\end{aligned}
$$

where $h=h_{\perp} / \cos \alpha$ is the critical slab depth for basal crack propagation. It can be shown that this critical slab depth $h$ goes through a minimum for a slope angle $\alpha=$ $39.2^{\circ}$, as illustrated in Fig. 2.

This calculation implicitly assumes (Fig. 1) that the slab depth is smaller than the crack size. Taking for $K_{I I c}$ a value of $1 \mathrm{kPa} \sqrt{m}$ (3) (which is not likely to be true in shear, as mentioned above), the critical slab depth for a snow density of $400 \mathrm{~kg} / \mathrm{m}^{3}$ and a slope of $40^{\circ}$ should be about $15 \mathrm{~cm}$, to be compared to crack sizes of some tens of meters, which should validate the approximation. If instead we consider that the actual shear toughness is significantly larger, as argued above, the critical depth should be accordingly increased. It may be of the order of $5 \mathrm{~m}$ for a shear toughness of $17.2 \mathrm{kPa} \sqrt{m}$ and a slope of $40^{\circ}$, and even more for different slope angles (see Fig. 2), which may not be small as compared to reasonable basal crack sizes. In this case, the approximation $h / E_{1} \ll a_{s} / E_{2}$ is no longer valid: energy should be considered as stored in a 3-D volume, and Griffith's criterion may be used. 




(a)

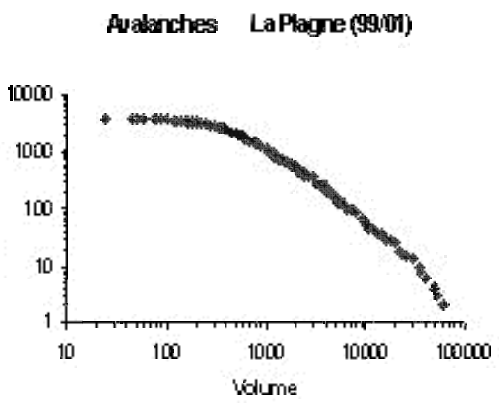

(b)
Fig. 3. Distribution of avalanche frequency vs. duration of the associated acoustic emission from Val Frejus data (a) or vs. avalanche size (slab depth $x$ crown crack length) from La Plagne data (b): the data obey a power law up to a cutoff associated with the spatial scale of the system.

\section{Population of elastically interacting defects}

The last reason that may affect the applicability of Griffith's criterion is that the snow cover, and in particular, the weak layer, are likely to contain a population of elastically interacting cracks instead of a single crack. Since artificial avalanche triggering is likely to be controlled by the stability of a single major basal crack formed under local and rapid external loading (e.g. a skier), the detailed structure and influence of preexisting cracks may be ignored as a first approximation, or taken into account considering the multi-cracked snow cover as a continuous average medium (mean field approach). By contrast, the knowledge of the collective behaviour of such a population of elastically coupled cracks is probably essential in the description of natural triggerings. Though triggering conditions in this situation may be, in principle, deterministically computed in each particular case, this task is untractable in practice. This kind of complex system that is governed by nonlinear deterministic equations may exhibit a chaotic behaviour, also often associated with scale invariant fractal structures (e.g. Turcotte, 1997). Scale invariance means that if $m(x)$ is the measure of a physical quantity at a scale $x$, the measure of the same quantity at a scale $\lambda x$ is:

$m(\lambda x)=\lambda^{\alpha} m(x)$,

where $\alpha$ is the scaling law exponent. The only functional that obeys Eq. (11) is a power law:

$m(x)=K x^{\alpha}$.

This is why, for instance, frequency/size or frequency/duration distributions in these fractal scale invariant systems obey power laws. There are a number of examples of such systems in geophysics, for instance in earthquakes, volcanic eruptions, rock falls or landslides.

Though snow avalanches are likely to behave in a similar way, as far as we know, the validity of fractal statistics has never been tested on these systems so far. The major problem is to find data that are both meaningful and available. A particular difficulty is linked to the fact that many avalanches are released during snow storms and are not visually recorded; this may bias the statistics, which is why, as a starting point, we analysed the distribution of the avalanche frequency as a function of the duration of acoustic emission, obtained from data recorded in the Valfréjus ski resort in the French Alps (6). Despite the fact that the results displayed in Fig. 3a were obtained from different avalanche channels, the distribution clearly obeys a single power law with a critical exponent close to 1.6. In a similar way, Fig. $3 b$ shows the cumulative distribution of avalanches vs. their size (defined as the product of the slab depth by the crown crack length), also obtained from different gullies in the La Plagne ski resort. We also obtain a power law with a critical exponent of 1.4. As usual, the cutoff at large durations or volumes is imposed by the largest dimension of the system, the scatter in the cutoff value probably resulting from the different sizes of the channels in which avalanches were recorded. However, the critical exponent obtained for each type of physical quantity (duration, size, etc.) is independent of the local topography. This universal character, if confirmed, may help in the statistical prediction of avalanches of given amplitudes. In addition, the determination of such a critical exponent is of interest, in order to understand the physics of avalanche triggerings: discrete elements and cellular automata simulations are in progress, in order to simulate avalanches of interacting granular objects or resulting from coalescence of interacting cracks. The comparison of the corresponding critical exponents with the experimental ones will allow us to validate or invalidate the physics of interactions introduced in the simulations.

\section{Conclusions}

The application of Griffith's criterion to slab avalanche triggerings suffers from inconsistencies in toughness data. We showed that some of these inconsistencies may be solved considering that snow shear toughness has a significantly larger value than that obtained from tensile toughness through elasticity calculations, due to rapid healing of broken ice bonds. Some deviations from Griffith's criterion may also occur when a significant part of the elastic energy is stored in a shallow slab, leading to a reduction of basal crack size values in the critical triggering situation. Nevertheless, this criterion remains a reasonable approach for artificially triggered avalanches. In contrast, we suggest that avalanches 
resulting from failure of multi-cracked weak layers, and in particular, natural avalanches, are more likely to be described using the theory of critical phenomena. We show indeed for the first time that both frequency/acoustic emission duration and frequency/size plots obtained from field data obey scale invariant power law distributions. This result provides a basis for an extensive study of triggering processes using discrete elements and cellular automata simulations.

Acknowledgements. A. Duclos (Transmontagne), and Valfréjus and La Plagne ski resorts are acknowledged for kindly providing field data on avalanche acoustic emission and sizes, H. Kirchner for helpful remarks on toughness values, and the "Conseil Général de l'Isère" for financial support through the "Pôle Grenoblois des Risques Naturels".

\section{References}

Gibson, L. J. and Ashby, M. F.: Cellular Solids: Structure and Properties, Pergamon Press, Oxford, 1987.

Kirchner, H. O. K., Michot, G., and Suzuki, T.: Fracture toughness of snow in tension, Phil. Mag. A 80, 5, 1265-1272, 2000.

Louchet, F.: Creep instability of the weak layer and natural slab avalanche triggerings. Cold Regions Science and Technology, 33, 141-146, 2000.

Louchet, F.: A transition in dry snow slab avalanche triggering modes, Proc. Int. Symp. on snow, avalanches and impact on the forest cover, Innsbruck, 22-26 May 2000, Ann. of Glaciology, 32, 285-289, 2001.

Turcotte, D. L.: Fractals and Chaos in Geology and Geophysics, Cambridge University Press, 1997. 